\title{
AUTOMATIC ANESTHESIA CONTROL SYSTEM
}

\author{
Chandana R \\ Department of Medical Electronics \\ BMS College of Engineering, Bangalore \\ Gajalakshmi M \\ Department of Medical Electronics \\ BMS College of Engineering, Bangalore
}

\author{
Kaveen V \\ Department of Medical Electronics \\ BMS College of Engineering, Bangalore \\ Dr. Joshi Manisha S \\ Professor \\ Department of Medical Electronics \\ BMS College of Engineering, Bangalore
}

\begin{abstract}
It is important that during any operation, for the patient to be in an anesthetic condition. The patient won't feel any pain during the medical procedure using anesthetics and the impact of the anesthesia should be there for as long as the operation goes on. It is also important to measure biomedical parameters such as heart rate and body temperature since these parameters play a vital role. It will create serious health problems if proper dosage of anesthesia is not administered at specified time intervals and to overcome such unfavorable situations this project has been designed to develop an automatic anesthesia control system. Using this, the predefined number of doses can be administered at regular time intervals by simultaneously measuring parameters such as heart rate and temperature.
\end{abstract}

Keywords - anesthesia, heart rate, body temperature

\section{INTRODUCTION}

In order to control pain and stress for the patient undergoing surgery, anesthesia plays a vital role. In the hospitals when any major operation is performed, the patient must be in anesthetized condition. For operations going on for a long time, complete dose cannot be given at a single stroke since it might lead to death of the patient and if less dosage is given, the patient may wake up in the middle of the operation. To avoid such situations, anesthesia must be provided at regular time intervals for as long as the operation goes on. It is also important to measure biomedical parameters such as heart rate and body temperature since these parameters play a vital role and define the overall condition of the patient. To overcome such problems the design of an automatic anesthesia control system is effective. This is a microcontroller based system. In recent times due to rapid advancement of embedded systems in real time computing, having the ability to control many devices at a time, these embedded systems paved the path to the invention of microcontrollers. The number of doses is calculated in prior and given as a predefined value. The doses are injected at regular intervals of time by simultaneously monitoring the parameters such as heart rate and temperature.

\section{PROPOSED SYSTEM}

\section{A. AIM}

It is important that during any operation, for the patient to be in an anesthetic condition. The patient won't feel any pain during the medical procedure using anesthetics and the impact of the anesthesia should be there for as long as the operation goes on. It is also important to measure biomedical parameters such as heart rate and body temperature since these parameters play a vital role. It will create serious health problems if proper dosage of anesthesia is not administered at specified time intervals and to overcome such unfavorable situations this project has been designed to develop an automatic anesthesia control system. The project aims at injecting the specified number of doses of anesthesia while simultaneously monitoring the clinical parameters such as heart rate. Additionally, temperature parameters are checked by corresponding sensors.

\section{B. PROPOSED SYSTEM}

In the proposed system microcontroller based system is used to inject the predefined number of anesthesia doses to the patient at regular time intervals. The dose of anesthesia must be known in advance, as a predefined value is programmed as input. The microcontroller is programmed using an embedded system to regulate the dose of anesthesia. We also measure the biomedical parameters such as heart rate and temperature since the measurement of biomedical parameters is a vital process. These parameters determine the overall condition of the patient, only based on these parameters the movement of the stepper motor is determined.

\section{SOFTWARE IMPLEMENTATION}

The software implementation is done using Proteus Software. Proteus is a simulation and design software tool developed by Labcenter Electronics for Electrical and Electronic circuit design. It is a software suite containing schematic, simulation as well as PCB designing. ISIS is the software used to draw schematics and simulate the circuits in real time. The simulation 
allows human access during run time, thus providing real time simulation.

Components used in the software design are

1. Microcontroller - Arduino Uno - to control the overall operation

2. Temperature Sensor - LM35 - to measure body temperature

3. Heart Beat Sensor to measure heartbeat

4.Stepper Motor to control the movement of the Syringe Infusion Pump

6. Motor driver - L293D - to control de motor

7. LCD Display - LM016L - to display the condition of the parameters.

1 .

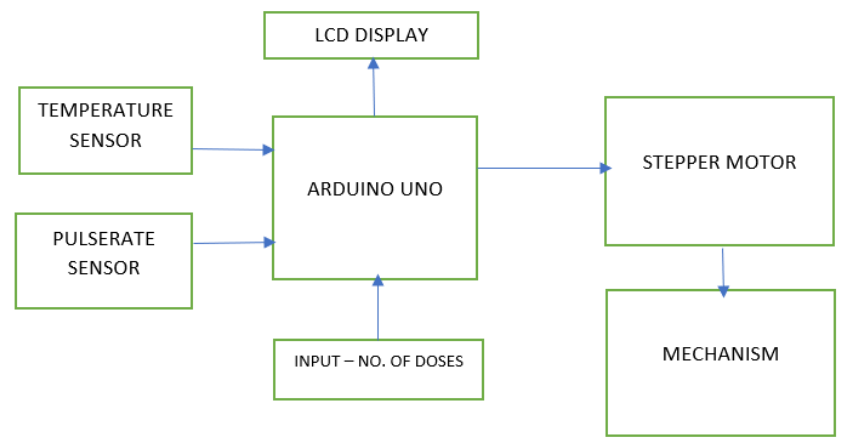

Fig. 1. Block Diagram

\section{MECHANISM}

- $\quad$ Run the AUTOMATIC ANAESTHESIA CONTROL SYSTEM simulation.

- The number of doses to be administered is given as input.

- The first dose is injected.

- It then checks for the inputs from the sensors and varies if the heartbeat is normal.

- If the heartbeat is normal, the next dose is injected or else, if it is not normal, then it alerts the doctor by displaying on the LCD.

- This process will be repeated for the specified number of doses.

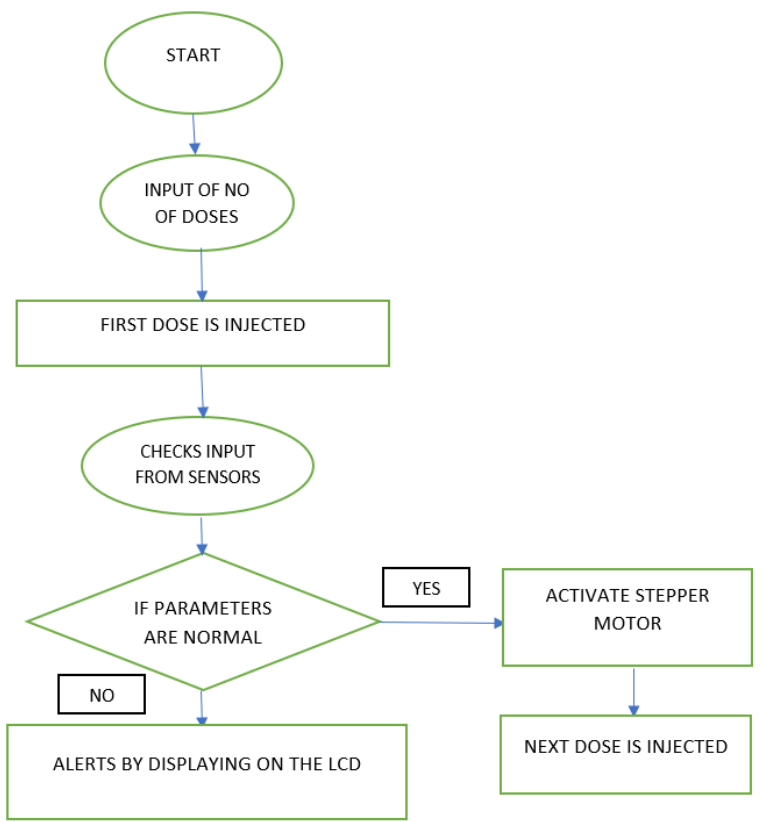

Fig. 2.Flowchart

\section{EXPERIMENT AND RESULT}

The proposed system takes the number of doses as the input and parameters such as heart rate and temperature are monitored. The movement of the stepper motor is determined by the conditions of heart rate. If the heart rate is normal, the stepper motor moves and it is displayed so on the LCD screen. If the heart rate falls below or above the normal range, the stepper motor does not rotate and the doctor is alerted by displaying on the LCD screen. Additionally, temperature parameters are also monitored.

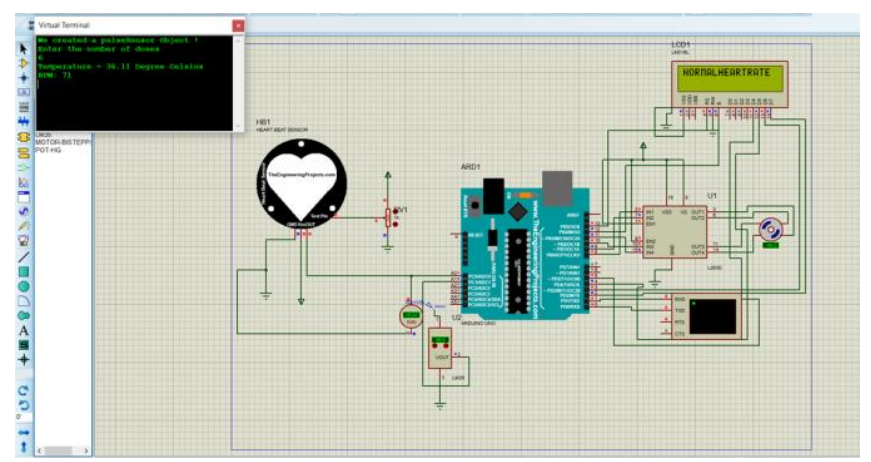

Fig. 3. Proteus Implementation case for normal heart rate 


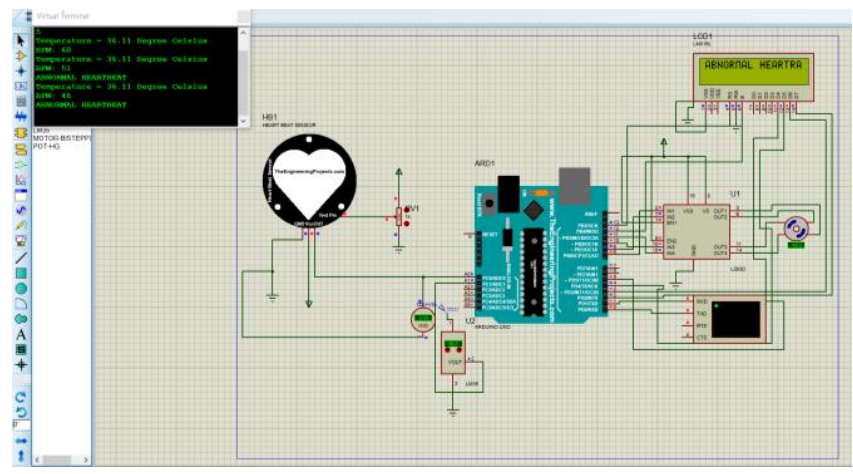

Fig. 4. Proteus Implementation case for abnormal heart rate

\section{CONCLUSION}

In the proposed system microcontroller based system is used to inject the predefined number of anesthesia doses to the patient at regular time intervals. The dose of anesthesia must be known in advance, as a predefined value is programmed as input. The microcontroller is programmed using an embedded system to regulate the dose of anesthesia. We also measure the biomedical parameters such as heart rate and temperature since the measurement of biomedical parameters is a vital process. These parameters determine the overall condition of the patient, only based on these parameters the movement of the stepper motor is determined.

\section{Advantages}

- The proposed system does not require the physical presence of an anesthetist to monitor the doses of anesthesia to be given during the surgery.

- This also increases the precision as it eliminates human error involved.

- It measures the biomedical parameters as this plays a vital role in determining the overall condition of the patient

\section{ACKNOWLEDGEMENT}

During the completion of this project many people provided help and support when required. We would like to acknowledge them. Firstly we would like to thank our HOD Dr. K Vijaylakshmi for providing us with the opportunity to work on this project. We would also like to thank our guide Dr. Joshi Manisha S for her guidance and valuable advice at every single stage of the project. We would like to thank them for constantly motivating us to work harder.

\section{SCOPE FOR FUTURE WORK}

This project can be further developed by taking inputs for static parameters of the patient based on which the anesthesia doses are calculated and storing the drug dosage value for different modes of surgery in a database and hence calculating the number of doses.

\section{REFERENCE}

[1] Srinivasa Naidu.N ; Kavya Sai.M ; Meghana.P.V.S ; Sai Praneeth.M ; Manoj Kalyan.N . (2019) . Microcontroller Based Anaesthesia Injector . JASC: Journal of Applied Science and Computations, (Pg 2364 - 2373)

[2] Gokilavani.R ; Gokulapriya. M ; Jasmine Christy. A.R ; Jeeva. R. (2019) . ANESTHESIA CONTROL SYSTEM WITH MULTI SENSOR USING ARDUINO . International Journal of Innovative Research in Advanced Engineering (IJIRAE), (Pg 86 - 90)

[3] S.Krishnakumar ; J. Bethanney Janney ; W. Antony Josephine Snowfy; S. Joshin Sharon ; S. Vinodh Kumar . (2018) . Automatic anesthesia regularization system (AARS) with patient monitoring modules . International Journal of Engineering \&amp; Technology, ( Pg 48 - 52)

[4] Prashanth C ; Mohammed Salman ; Rohan K.R ; Govinda Raju. M ; Roopa. J. (2014) . Computerized Anesthesia Infusion System . International Journal of Electrical, Electronics and Computer Systems (IJEECS), (Pg - 2347-2820)

[5] Hanumant R.Vani , Pratik V, Makh, Mohanish \& Chandurkar.K . (2014) . Anesthesia Regularization using Heart Beat Sensor International Journal Of Engineering, Education And Technology (ARDIJEET), (Pg 1 - 9)

[6] Manikandan N, Muruganand S \& Vasudevan K . (2013) . Low Cost Anesthesia Injector Based On Arm Processor, International Journal of Advanced Research in Computer and Communication Engineering, (Pg 2810- 2813).

[7] Durgadevi S, Anbananthi . (2014) . Embedded System: Patient Life Secure System Based On Microcontroller. International Journal for Advance Research in Engineering and Technology, (Pg 142-147).

[8] Kraft HH \& Lees DE. (1984) . Closing the loop: How near is automated anesthesia?, Southern Med. J. ,(Pg 7-12).

[9] Vickers, MD, Morgan, M \& Spencer, PSS . (1991) . General Anaesthetics,7th edition, Butterworth Heinemann Ltd.,Oxford, (Pg 118-159).

[10] Vishnoi, R \& Roy, R.J. (1991) . Adaptive control of closed circuit anesthesia. IEEE Trans. Biomed. Eng., (Pg 39-47)

[11] Smt.Leela Salim , Abey Thomas , Akshay M , Athul K Alias, Muhammed Irshad E K . (2019) . Microcontroller based Anesthesia Injector . International Research Journal of Engineering and Technology (IRJET), (Pg 826 - 829)

[12] P. Deepakfranklin and M. Krishnamoorthi . (2018) . Monitoring Multiple Biomedical Parameters to Automate Anesthesia Injector Using FPGA . International Conference on Computer, Communication, and Signal Processing (ICCCSP), pp. 1-5, doi: 10.1109/ICCCSP.2018.8452862. 